\title{
PREVALENCIA DE CORIOAMNIONITIS HISTOLÓGICA EN PACIENTES CON TRABAJO DE PARTO PRETÉRMINO ESPONTÁNEO. POPAYÁN, COLOMBIA, 2014-2016
}

\section{Prevallence of histologic chorioamnionitis in patients with spontaneous pre-term labour in Popayán, Colombia, 2014-2016}

Carolina Arias-Peláez, $M D^{I}$; Cristal Ximena Gallego-Betancourt, $M D^{I}$; Geovanni Alexander Mera-Ijaji, $\mathrm{MD}^{1}$; Roberth Alirio Ortiz-Martínez, MD, $\mathrm{MSc}^{2}$; José Enrique Chagüendo-García, $M D^{3}$

Recibido: julio 27/16 - Aceptado: mayo 8/17

\section{RESUMEN}

Objetivo: determinar la prevalencia de la corioamnionitis histológica en gestantes con trabajo de parto pretérmino espontáneo, evaluar posibles factores de riesgo y la asociación con signos clínicos y paraclínicos.

Materiales y métodos: estudio descriptivo de prevalencia, con análisis secundario exploratorio en gestantes con embarazo menor a 37 semanas de gestación, con parto espontáneo, en quienes se dispuso de sus placentas para estudio histopatológico, durante un periodo de 2 años, en un hospital de alta complejidad en Popayán, Colombia. Se excluyeron partos pretérmino secundarios. Muestreo consecutivo, con tamaño muestral de 166 gestantes; se midieron variables sociodemográficas, clínicas,

1 Residentes de Ginecología y Obstetricia, Universidad del Cauca, Popayán (Colombia).Ximegabe@gmail.com

2 Magíster en Epidemiología, ginecoobstetra; docente Universidad del Cauca, Popayán (Colombia).

3 Ginecoobstetra; docente Universidad del Cauca, Popayán (Colombia). de laboratorio e imágenes diagnósticas, y resultado histopatológico de la placenta. En el análisis se estimó la prevalencia de periodo de corioamnionitis histológica. Se obtuvo la razón de prevalencias como medida de asociación y el OR diagnóstico (ORD) con su respectivo intervalo de confianza al 95\%.

Resultados: en un periodo de 2 años se analizaron 160 gestantes y sus placentas, 110 placentas fueron reportadas como positivas para corioamnionitis histológica con una prevalencia de 68,75\% (IC 95\%: 61,49-76,00), esta fue más prevalente en gestaciones menores de 34 semanas ( $R P=1,48$; IC $95 \%$ : 1,201,83). En cuanto a la asociación de los síntomas y signos se encontró asociación significativa con la fiebre $(\mathrm{ORD}=4,7$; IC $95 \%$ : 1,05-21,09), la taquicardia materna $(\mathrm{ORD}=4,22$; IC $95 \%: 1,81-9,81)$, la taquicardia fetal (ORD = 3,74; IC $95 \%$ : 1,23-11,35), movimientos respiratorios ausentes $(\mathrm{ORD}=5,16$; IC $95 \%$ : 1,43-18,60), lago de líquido amniótico menor a $2 \mathrm{~cm}(\mathrm{ORD}=5,67$; IC $95 \%$ : 1,24-25,98), y presencia de neutrofilia (ORD = 2,97; IC $95 \%$ : 1,44-6,12). 
Conclusiones: la prevalencia de corioamnionitis histológica es del $67 \%$ en los partos pretérmino y varió en función de la edad gestacional.

Palabras clave: trabajo de parto pretérmino, corioamnionitis histológica, estudio de corte transversal.

\section{ABSTRACT}

Objective: To determine the presence of histologic chorioamnionitis in pregnant women in spontaneous pre-term labour, and to evaluate potential risk factors and association with clinical and paraclinical signs and symptoms.

Materials and methods: Descriptive prevalence study with secondary exploratory analysis in pregnant women under 37 weeks of gestation with spontaneous delivery in whom the placenta was used for histopathology testing, conducted over a 2-year period in a high complexity hospital in Popayán, Colombia. Secondary pre-term deliveries were excluded. Consecutive sampling, with a sample size of 166 pregnant women. Social, demographic, clinical, laboratory and imaging variables were measured, as well as the placental histopathology test result. The analysis estimated the prevalence of histological chorioamnionitis period. The prevalence ratio as a measure of association and the diagnostic OR (DOR) with its respective $95 \%$ confidence interval were obtained.

Results: Overall, 160 pregnant women and their placentas were analyzed over a 2-year period. There were positive reports for 110 placentas with histological chorioamnionitis, for a prevalence of $68.75 \%$ (95\% CI: 61.49-76.00), the prevalence being higher in gestations under 34 weeks $(\mathrm{PR}=1.48 ; 95 \%$ CI: 1.20-1.83). Regarding association of signs and symptoms, a significant association was found with fever $(\mathrm{DOR}=4.7$; $95 \% \mathrm{CI}$ : 1.05-21.09), maternal tachycardia $(\mathrm{DOR}=4.22 ; 95 \% \mathrm{CI}: 1.81-9.81)$, foetal tachycardia $(\mathrm{DOR}=3.74 ; 95 \% \mathrm{CI}: 1.23-11.35)$, absent breathing movements $(\mathrm{DOR}=5.16 ; 95 \%$ CI: 1.43-18.60), amniotic fluid lower than $2 \mathrm{~cm}$
$(\mathrm{DOR}=5.67 ; 95 \% \mathrm{CI}: 1.24-25.98)$, and presence of neutrophilia (DOR $=2.97 ; 95 \%$ CI: 1.44-6.12). Conclusions: The prevalence of histological chorioamnionitis was $67 \%$ in pre-term deliveries and varied in accordance with gestational age.

Key words: Pre-term labour, histological chorioamnionitis, cross-sectional study.

\section{INTRODUCCIÓN}

El nacimiento pretérmino es aquel que ocurre antes de las 37 semanas de gestación, y es una de las principales causas de morbi-mortalidad infantil y de morbilidad en la vida adulta (1-5), responsable de alrededor de un $70 \%$ de la mortalidad perinatal; cada año nacen 15 millones de recién nacidos prematuros, de los cuales 1,1 millones mueren como consecuencia de la inmadurez orgánica y funcional $(6,7)$, con implicaciones de índole social, familiar y económica. Se considera que los gastos anuales por su atención en Estados Unidos son alrededor de 25 billones de dólares (6). Su frecuencia varía siendo más frecuente en países de ingresos medios y bajos. Se estima una prevalencia global del 9,6\%; por regiones, un 8,1\% en América Latina y el Caribe; 6,2\% en Europa; 10,6\% en Norte América. En Colombia, la frecuencia oscila entre 10 a $12 \%$ (7). Una de sus causas es la corioamnionitis (8-10), en la que se debe distinguir entre la corioamnionitis clínica (CC) y la histológica $(\mathrm{CH})$; en la CC clásicamente su diagnóstico es clínico y se basa en los criterios expuestos por Gibbs (11, 12); en la $\mathrm{CH}$, menos estudiada, hay un proceso inflamatorio agudo caracterizado por infiltración de neutrófilos en cada una de las estructuras de la placenta; cuando afecta al corión y al amnios, se denomina corioamnionitis aguda; si afecta al velloso, se llama villitis aguda; si el proceso involucra el cordón umbilical se denomina funisitis (13). La contrapartida en el feto es el síndrome de respuesta inflamatoria fetal $(13,14)$.

La palabra "histológico" se ha introducido para especificar las diferencias entre el síndrome clínico (CC) y el diagnóstico patológico $(\mathrm{CH})$, estos tér- 
minos no son sinónimos y la confusión se origina cuando se utilizan indistintamente (13). Por otra parte, el diagnóstico de la $\mathrm{CH}$ cobra importancia si se tiene presente que ha sido asociado como factor de riesgo para resultados perinatales adversos, incluso parálisis cerebral (10, 15-18). La prevalencia de $\mathrm{HC}$ está en función de la edad gestacional (EG): a menor EG, mayor su prevalencia (9). Se ha descrito que la prevalencia puede llegar al 94,4\% en menores de 24 semanas (19), sin embargo, hay controversia al respecto.

La prueba de elección ante parto de la corioamnionitis es el estudio de líquido amniótico (LA) con cultivo obtenido por medio de amniocentesis, con el inconveniente de que es invasivo y, por tanto, no inocuo (20); la tinción de Gram del LA (21), la concentración de glucosa, los niveles de deshidrogenasa láctica (DHL) y la estearasa de leucocitos son utilizados para evaluar la infección en cavidad amniótica debido a su bajo costo y fácil disponibilidad (22-25). Otro marcador es la proteína C reactiva (PCR) que, aunque no está establecida dentro de los criterios de Gibbs, su uso ha adquirido una gran importancia en su diagnóstico $(26,27)$. Se han descrito marcadores ecográficos tales como: acortamiento cervical, presencia de sludge y el perfil biofísico fetal (PBF) (28), este último es un buen predictor de infección cuando el intervalo entre su realización y el parto es $<24$ horas (29-31). El enfoque de la mayor parte de las investigaciones en marcadores es en su rendimiento diagnóstico para la $\mathrm{CC}$ y son pocas en la $\mathrm{CH}$.

Teniendo en cuenta que hay pobre información sobre la prevalencia de $\mathrm{CH}$ en nuestro medio, y debido al comportamiento de los parámetros clínicos y paraclínicos en la $\mathrm{CH}$, se planteó este estudio con el objetivo de determinar la prevalencia de $\mathrm{CH}$ en pacientes con trabajo de parto pretérmino espontáneo en el Hospital Universitario San José (HUSJ) de Popayán, y evaluar posibles factores sociodemográficos de riesgo y la asociación con signos clínicos y paraclínicos.

\section{MATERIALES Y MÉTODOS}

Se realizó un estudio descriptivo de prevalencia, en el que se incluyeron gestantes con parto pretérmino espontáneo menor a 36 semanas 6 días, con producto único vivo, que consultaron desde el 1 de julio 2014 al 16 de mayo del 2016, en un hospital público de alta complejidad (HUSJ), centro de referencia del departamento del Cauca y sur occidente de Colombia, que atiende población del aseguramiento contributivo y subsidiado, en el sistema de seguridad social vigente en el país, en quienes se dispuso de sus respectivas placentas. Se excluyeron los partos pretérmino secundarios y las malformaciones congénitas mayores. Para el tamaño de muestra se utilizó la fórmula: $\mathrm{n}=\left(\mathrm{p}^{*} \mathrm{q}\right)$ $/(\mathrm{E} / 1,96)^{2}$, prevalencia mínima esperada del $12 \%$ $(9,16)$, margen de error tolerando del $5 \%$, nivel de confianza del $95 \%$, ajuste por no respuesta del $10 \%$, corrección por población finita y base poblacional de 1900 nacimientos en el año previo al estudio, con un tamaño final de 166 gestantes. Se realizó muestreo no probabilístico consecutivo.

Procedimiento. En mujeres con diagnóstico de parto pretérmino espontáneo estimado a partir de la ecografía del primer trimestre, o por el examen físico realizado al neonato por el pediatra al nacimiento, se les solicitó autorización para participar en el estudio mediante firma del consentimiento informado. Una vez se autorizó su ingreso al estudio se procedió a la obtención de la placenta colocándola en un recipiente de plástico con formol al 10\%, taponado en proporción de 1:20, debidamente identificado, el cual se trasladó el mismo día al servicio de patología del HUSJ, donde se realizó el siguiente protocolo para procesamiento de placentas: inicialmente se asignó un código de identificación y posteriormente se examinó la placenta. Primero las membranas, luego el cordón y por último las superficies fetal y materna. Se realizaron cortes de cordón transversalmente y de placenta, se tomaron cortes de 2-3 centímetros desde el inicio de las membranas en el punto donde se rompen hasta 
el margen placentario, luego se fijaron durante 24 horas; después de los procesos de posfijación y corte se procedió con la tinción con hematoxilina-eosina, luego se entregó al patólogo para su lectura, el cual desconocía el motivo del estudio placentario. El diagnóstico de $\mathrm{CH}$ se dio por la identificación de leucocitos polimorfonucleares de origen materno en las membranas ovulares, placa corial y cordón $(13,32,33)$.

Después de la recolección de la placenta se procedió con la entrevista a las pacientes por personal entrenado, mediante un cuestionario semiestructurado, diseñado por los investigadores, revisado por expertos y ajustado por prueba piloto, también se tomó información de la historia clínica institucional. Se elaboró una base de datos en Excel realizando el control de ingreso de estos a través de reglas de validación a fin de garantizar la confiabilidad y calidad de la información; posteriormente, se llevó al programa Stata versión 10.0, en el que se efectuó el análisis.

Se midieron las siguientes variables: edad materna; edad gestacional, que se estratifico en $>$ y $<$ de 34 semanas, debido a que según estudios la prevalencia de $\mathrm{CH}$ es mayor a menor EG $(15,16$, 34-39); zona de residencia, tipo de unión, etnia (blanca y mestiza como referencia y negra e indígena como riesgo), tipo de aseguramiento (contributivo, subsidiado), estrato socioeconómico (ESE, de referencia 3, 4 y 5, y de riesgo el 1 y 2), atención prenatal adecuada (número de controles $>3$ ), y EG de inicio del control en el primer trimestre; fórmula obstétrica, historia de parto pretérmino, infección urinaria diagnosticada por urocultivo, número de tactos vaginales ( $>6$ como riesgo), actividad uterina refractaria (progresión del parto a pesar de manejo). Se evaluaron como pruebas diagnósticas: fiebre $\left(>38^{\circ} \mathrm{C}\right)$, taquicardia materna (>100 lat/min), taquicardia fetal (> $160 \mathrm{lat} / \mathrm{min})$, PCR anormal ( $>6 \mathrm{mg} / \mathrm{dl}$ ), hemograma (leucocitos $>15000$ y neutrófilos $>80 \%$ como alterados), características del líquido amniótico (LA) (fétido o no fétido), perfil biofísico fetal (PBF) (se analizó cada componente: tono, volumen del LA, movimientos respiratorios y gruesos), monitoreo fetal (normal la categoría I y anormal categorías II y III).

Análisis estadístico. Para determinar la prevalencia de $\mathrm{CH}$ se tomó como numerador la población con el estudio histopatológico positivo y como denominador el total de gestantes con parto pretérmino espontáneo, en las que se dispuso del estudio histopatológico. Las variables se analizaron individualmente, desde el punto de vista exploratorio, para mirar la normalidad de su distribución e identificar valores extremos y perdidos que pudieran incidir en el resultado; posteriormente se comparó el grupo de gestantes con parto pretérmino y $\mathrm{CH}$ con aquellas con parto pretérmino sin $\mathrm{CH}$. Se obtuvo como medida de asociación la razón de prevalencia (RP) y su respectivo intervalo de confianza (IC) al $95 \%$ para determinar la frecuencia de exposición entre los grupos con y sin $\mathrm{CH}$, además se estimaron los Odds Ratio Diagnóstico (ORD) para los parámetros paraclínicos y sus respectivos IC al $95 \%$. Se utilizó la prueba $t$ de Student para variables continuas con distribución normal previo análisis de varianza, para las de distribución no normal se utilizó la U de Mann-Whitney previa aplicación de test de normalidad de Shapiro Wilk y chi cuadrado o Fisher según correspondiera.

El estudio recibió el aval del comité de ética del HUSJ. Se garantizó la confidencialidad de la información.

\section{RESULTADOS}

En el periodo de estudio se atendieron un total de 3.385 nacimientos (julio-diciembre de 2014: 882; enero-diciembre de 2015: 1.714, y enero-mayo de 2016: 789); de estos, un total de 172 (5,1\%) partos pretérmino espontáneos, en 12 de ellos no se dispuso de sus placentas por lo que se excluyeron, quedando al final 160 para análisis. De estas 160 placentas se encontró $\mathrm{CH}$ en 110, obteniendo una prevalencia de 68,75\% (IC $95 \%$ : 61,49-76,00). 


\begin{tabular}{|c|c|c|c|}
\hline \multicolumn{4}{|c|}{$\begin{array}{l}\text { Tabla } 1 . \\
\text { Características basales de las gestantes con parto pretérmino en el HUSJ, } \\
\text { Popayán, Colombia, julio 2014-mayo } 2016\end{array}$} \\
\hline Características & $\begin{array}{l}\text { Corioamnionitis } \\
\qquad n=110\end{array}$ & $\begin{array}{l}\text { No corioamnionitis } \\
\qquad n=50\end{array}$ & valor $\mathbf{p}$ \\
\hline Edad (media) $( \pm \mathrm{DE})^{*}$ & $23,7( \pm 7,05)$ & $23,42( \pm 6,89)$ & $0,81^{\dagger}$ \\
\hline \multicolumn{4}{|l|}{ Gravidez } \\
\hline Nulípara & $48(43,13)$ & $21(42,00)$ & $0,84 \div$ \\
\hline Multíparas & $62(56,36)$ & $29(58,00)$ & \\
\hline \multicolumn{4}{|l|}{ Etnia } \\
\hline Blanco/Mestizo & $83(75,45)$ & $39(78,00)$ & $0,72 \ddagger$ \\
\hline Negro/Indígena & $27(24,55)$ & $11(22,00)$ & \\
\hline \multicolumn{4}{|l|}{ Estrato socioeconómico } \\
\hline Bajo & $85(77,27)$ & $41(82,00)$ & $0,49 \ddagger$ \\
\hline Medio & $25(22,73)$ & $9(18,00)$ & \\
\hline \multicolumn{4}{|l|}{ Tipo de unión } \\
\hline Estable & $78(70,91)$ & $30(60,00)$ & $0,17 \ddagger$ \\
\hline No estable & $32(29,09)$ & $20(40,00)$ & \\
\hline \multicolumn{4}{|l|}{ Aseguramiento } \\
\hline Contributivo & $21(19,09)$ & $9(18,00)$ & $0,87 \ddagger$ \\
\hline Subsidiado & $89(80,91)$ & $41(82,00)$ & \\
\hline \multicolumn{4}{|l|}{ Residencia } \\
\hline Urbano & $85(77,27)$ & $42(84,00)$ & $0,33^{\ddagger}$ \\
\hline Rural & $25(22,73)$ & $8(16,00)$ & \\
\hline
\end{tabular}

Fuente: datos del estudio.

Los valores se expresan en (\%).

${ }^{*}$ DE: desviación estándar.

${ }^{\dagger} t$ de Student.

$¥$ Chi cuadrado.

Respecto a las características basales, la edad materna, la gravidezy las características sociodemográficas no mostraron diferencias (tabla 1). Respecto a los antecedentes clínicos, la $\mathrm{CH}$ fue más prevalente en el grupo de $\mathrm{EG}<34$ semanas $(\mathrm{RP}=1,48$; IC $95 \%: 1,20-1,83)$, las otras variables analizadas presentaron similares RP entre los grupos (tabla 2).

En cuanto a la asociación de los síntomas y signos se encontró asociación significativa entre la fiebre, la taquicardia materna, la taquicardia fetal, movimientos respiratorios ausentes, lago de LA menor a 2 y la presencia de neutrofilia (tabla 3 ).

\section{DISCUSIÓN}

Nuestro estudio encontró una prevalencia de $\mathrm{CH}$ del $68 \%$. Se encontró asociación con la edad gestacional menor a 34 semanas y asociación con síntomas generales maternos, disminución de los 


\begin{tabular}{|c|c|c|c|}
\hline \multicolumn{4}{|c|}{$\begin{array}{l}\text { Tabla } 2 . \\
\text { Factores de riesgo para corioamnionitis histológica, HUSJ Popayán, } \\
\text { Colombia, julio 2014-mayo } 2016\end{array}$} \\
\hline Posibles variables asociadas & $\begin{array}{l}\text { Corioamnionitis } \\
\text { Histológica }(n=110)\end{array}$ & $\begin{array}{l}\text { No Corioamnionitis } \\
\text { Histológica }(n=50)\end{array}$ & $\begin{array}{l}\text { Razón de } \\
\text { prevalencia } \\
\text { (IC } 95 \%)\end{array}$ \\
\hline \multicolumn{4}{|l|}{ Historia de parto pretérmino* } \\
\hline Sí & $7(11,29)$ & $2(6,89)$ & $1,15(0,79-1,69)$ \\
\hline No (Ref.) & $55(88,71)$ & $27(93,11)$ & - \\
\hline \multicolumn{4}{|l|}{ Edad gestacional } \\
\hline$<34$ semanas & $61(55,45)$ & $12(24,00)$ & $1,48(1,20-1,83)$ \\
\hline$>34$ semanas (Ref.) & $49(44,55)$ & $38(76,00)$ & - \\
\hline \multicolumn{4}{|l|}{ Control prenatal } \\
\hline No adecuado & $77(70,00)$ & $29(58,00)$ & $1,18(0,93-1,51)$ \\
\hline Adecuado (Ref.) & $33(30,00)$ & $21(42,00)$ & - \\
\hline \multicolumn{4}{|l|}{ IVU $^{\dagger}$ actual } \\
\hline Sí & $15(13,64)$ & $4(8,00)$ & $1,71(0,90-1,51)$ \\
\hline No (Ref.) & $95(86,36)$ & $46(92,00)$ & - \\
\hline \multicolumn{4}{|l|}{ Tactos vaginales } \\
\hline 6 o más & $10(9,09)$ & $2(4,00)$ & $1,23(0,93-1,62)$ \\
\hline < 6 (Ref.) & $100(90,91)$ & $48(96,00)$ & - \\
\hline
\end{tabular}

Fuente: datos del estudio.

Los valores se expresan en (\%).

* Se excluyeron pacientes nulíparas.

†IVU: infección de vías urinarias.

movimientos respiratorios en el perfil biofísico, del lago mayor de LA en el ultrasonido obstétrico y neutrofilia.

Respecto a la prevalencia, nuestros hallazgos son concordantes con investigaciones publicadas por Xie et al., quienes encontraron una frecuencia de $\mathrm{CH}$ en gestaciones comprendidas entre 28 a 33 semanas 6 días de 68,0\% (138/203) (15). Xie et al., en 371 gestantes menores de 34 semanas, reportan una prevalencia del $70 \%$ (16); Holzman et al. informan una prevalencia de $63 \%$ (34), al igual que Nowak et al., quienes muestran una frecuencia de $\mathrm{CH}$ de 73,7\% en menores de 35 semanas (35). En contraposición, otros informan prevalencias menores, como el estudio de Lee et al., que reportan una prevalencia del $24 \%$ en pretérminos entre 34 y 36 semanas 6 días (10); Romero et al. señalan que la prevalencia de la CH está dada en función de la EG al nacimiento (9); Xie et al. encontraron que la incidencia de $\mathrm{CH}$ varió con la EG, así: entre 28 y 29: 86,7\%, entre 30 y 31: 70,1\% y entre 32 y 33: 61,4\% (16).

En cuanto a los criterios diagnósticos, Curtin et al., en una cohorte retrospectiva reportan la presencia de fiebre en el 80,6\% de las pacientes con $\mathrm{CH}$, con un $\mathrm{OR}=4,63$ (IC95\%: 3,09-6,93), concluyendo que la fiebre es un predictor indepen- 


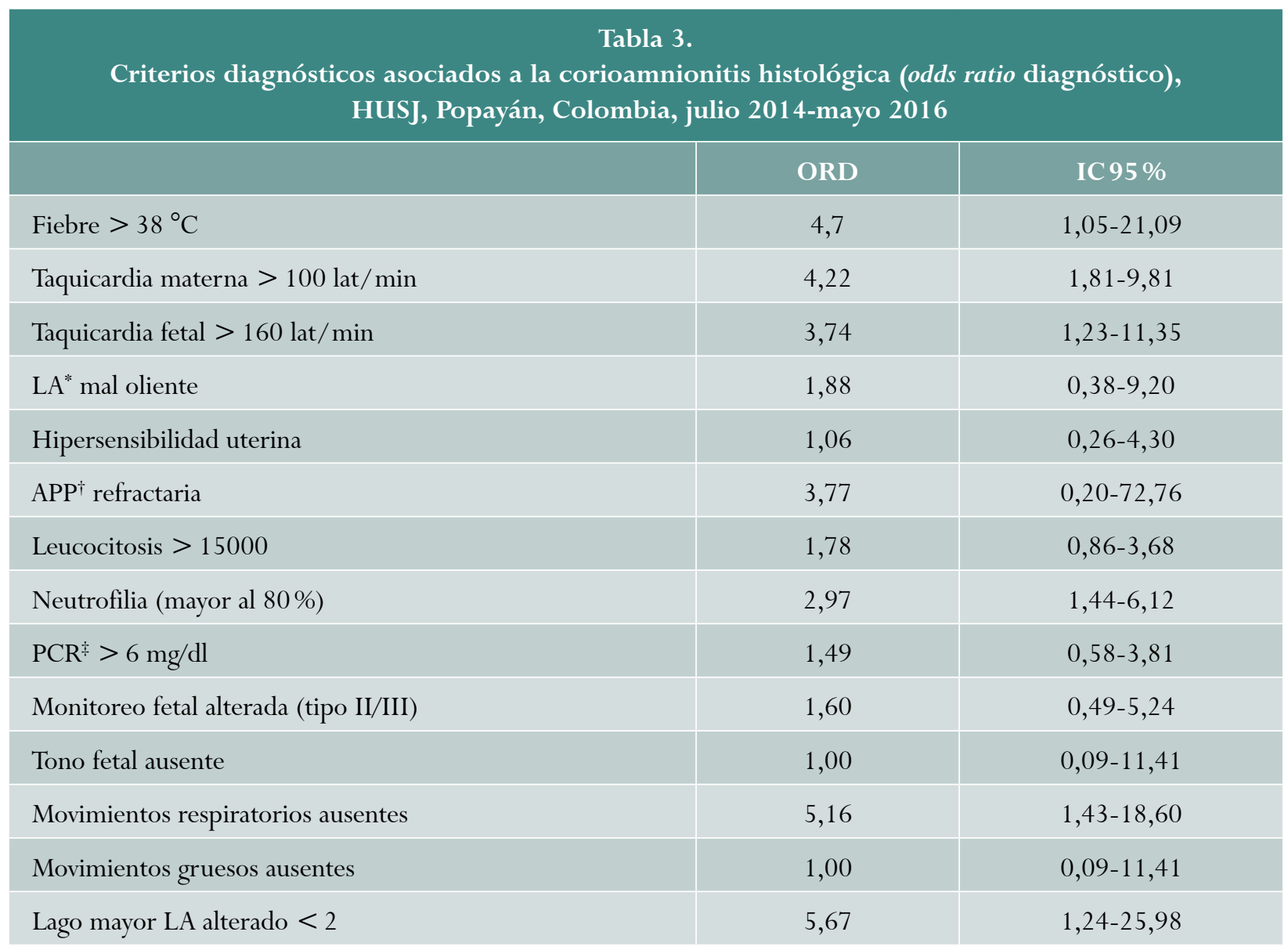

Fuente: datos del estudio.

* LA: líquido amniótico.

$\dagger$ APP: amenaza de parto pretérmino.

¥ PCR: proteína C reactiva.

diente de CH (40); otra cohorte retrospectiva con 641 placentas examinadas reporta, para fiebre, un $\mathrm{OR}=2,83$ (IC $95 \%: 1,76-4,55)$ (41); Heller et al. no encontraron diferencias significativas en la proporción de fiebre intraparto entre los grupos con y sin $\mathrm{CH}$ (42). Al analizar las variables del bienestar fetal, Curtin et al. reportan que no encontraron una asociación por análisis multivariado entre desaceleraciones variables severas y CH (41); otros sí reportan esta relación. Salafia et al. encontraron que las desaceleraciones variables severas de la frecuencia cardiaca fetal (FCF) ante parto se relacionaron directamente con $\mathrm{CH}(\mathrm{p}=0,012)$, al igual que en el trabajo de parto la disminución de la variabilidad de la FCF se relacionó con $\mathrm{CH}(\mathrm{p}=0,005)$ (43). Con respecto a la ausencia de movimientos respiratorios,
Sherer et al., en pretérminos menores de 32 semanas, reportan una mayor frecuencia de ausencia de movimientos respiratorios en vasculitis umbilical severa de $32 \%$ frente a $15 \%$, hallazgo este significativo ( $\mathrm{p}=0,049)$ (44); Ghidini et al., en menores de 32 semanas, no encontraron relación entre la inflamación placentaria aguda y la puntuación total del PBF o sus componentes individuales (45).

Como fortalezas del estudio se encuentran: la variable resultado fue estandarizada, los patólogos no conocían la sospecha diagnóstica, la EG fue confiable, los parámetros tomados en cuenta para el análisis fueron los más cercanos al nacimiento.

Como debilidades, al ser un estudio en un nivel de alta complejidad pudo presentarse un sesgo de referencia, ya que la población atendida en este 
nivel es la de más alto riesgo obstétrico; además, se presentaron 12 pérdidas (7\%) de las cuales no se dispuso de las placentas para sus estudio, lo cual puede llevar a una posible subestimación de la prevalencia. Por otra parte, una de las variables incluidas fue la infección urinaria y esta, por sí sola, podría explicar algunos de los hallazgos clínicos y paraclínicos.

\section{CONCLUSIÓN}

La prevalencia encontrada de $\mathrm{CH}$ fue alta y está asociada a la EG menor de 34 semanas. Se recomiendan estudios en este tópico y su relación con resultados neonatales.

\section{REFERENCIAS}

1. Moster D, Lie RT, Markestad T. Long-term medical and social consequences of preterm birth. N Engl J Med. 2008;359:262-73. https://doi.org/10.1056/ NEJMoa0706475.

2. Marlow N, Wolke D, Bracewell MA, Samara M, EPICure Study Group. Neurologic and developmental disability at six years of age after extremely preterm birth. N Engl J Med. 2005;352:9-19. https://doi. org/10.1056/NEJMoa041367.

3. Hille ET, den Ouden AL, Saigal S, Wolke D, Lambert M, Whitaker A, et al. Behavioural problems in children who weigh $1000 \mathrm{~g}$ or less at birth in four countries. Lancet. 2001;357:1641-3. https://doi.org/10.1016/ S0140-6736(00)04818-2.

4. Strauss RS. Adult functional outcome of those born small for gestational age: Twenty-six-year follow-up of the 1970 British Birth Cohort. JAMA. 2000;283:62532. https://doi.org/10.1001/jama.283.5.625.

5. Barker DJP, Forsen T, Uutela A, Osmond C, Eriksson JG. Size at birth and resilience to effects of poor living conditions in adult life: longitudinal study. BMJ. 2001;323:1273-6. https://doi.org/10.1136/ bmj.323.7324.1273.

6. Flood K, Malone FD. Prevention of preterm birth. Semin Fetal Neonatal Med. 2012;17:58-63. https:// doi.org/10.1016/j.siny.2011.08.001.
7. Bick D. Born too soon: The global issue of preterm birth. Midwifery. 2012;28:401-2. https://doi. org/10.1016/j.midw.2012.06.010.

8. Kim SM, Romero R, Park JW, Oh KJ, Jun JK, Yoon $\mathrm{BH}$. The relationship between the intensity of intra-amniotic inflammation and the presence and severity of acute histologic chorioamnionitis in preterm gestation. J Matern Fetal Neonatal Med. 2015;28:1500-9. https://doi.org/10.3109/1476705 8.2014.961009.

9. Kim CJ, Romero R, Chaemsaithong P, Chaiyasit N, Yoon BH, Kim YM. Acute chorioamnionitis and funisitis: definition, pathologic features, and clinical significance. Am J Obstet Gynecol. 2015;213(4 Suppl):S29-52. https://doi.org/10.1016/j. ajog.2015.08.040.

10. Lee SM, Park JW, Kim BJ, Park C-W, Park JS, Jun $\mathrm{JK}$, et al. Acute histologic chorioamnionitis is a risk factor for adverse neonatal outcome in late preterm birth after preterm premature rupture of membranes. PLoS One. 2013;8:e79941. https://doi.org/10.1371/ journal.pone.0079941.

11. Gibbs RS, Duff P. Progress in pathogenesis and management of clinical intraamniotic infection. Am J Obstet Gynecol. 1991;164:1317-26. https://doi. org/10.1016/0002-9378(91)90707-X.

12. Newton ER. Chorioamnionitis and intraamniotic infection. Clin Obstet Gynecol. 1993;36:795-808. https://doi.org/10.1097/00003081-19931200000004.

13. Redline RW, Faye-Petersen O, Heller D, Qureshi F, Savell V, Vogler C. Amniotic infection syndrome: Nosology and reproducibility of placental reaction patterns. Pediatr Dev Pathol. 2003;6:435-48. https:// doi.org/10.1007/s10024-003-7070-y.

14. Pacora P, Chaiworapongsa T, Maymon E, Kim YM, Gomez R, Yoon BH, et al. Funisitis and chorionic vasculitis: The histological counterpart of the fetal inflammatory response syndrome. J Matern Fetal Neonatal Med. 2002;11:18-25. https://doi. org/10.1080/jmf.11.1.18.25. 
15. Xie AL, Di XD, Chen XM, Hu YC, Wang YH. [Factors and neonatal outcomes associated with histologic chorioamnionitis after premature rupture of membranes in the preterms]. Zhonghua Fu Chan Ke Za Zhi. 2012;47:105-9.

16. Xie A, Zhang W, Chen M, Wang Y, Wang Y, Zhou $\mathrm{Q}$, et al. Related factors and adverse neonatal outcomes in women with preterm premature rupture of membranes complicated by histologic chorioamnionitis. Med Sci Monit. 2015;21:390-5. https://doi.org/10.12659/MSM.891203.

17. Grether JK, Nelson KB. Maternal infection and cerebral palsy in infants of normal birth weight. JAMA. 1997;278:207-11. https://doi.org/10.1001/ jama.1997.03550030047032.

18. Smulian J. Clinical chorioamnionitis and histologic placental inflammation. Obstet Gynecol. 1999;94: 1000-5. https://doi.org/10.1016/S0029-7844 (99)00416-0.

19. Russell P. Inflammatory lesions of the human placenta. III: The histopathology of villitis of unknown aetiology. Placenta. 1980;1:227-44. https://doi.org/10.1016/ S0143-4004(80)80005-1.

20. Romero R, Espinoza J, Gonçalves L, Kusanovic J, Friel L, Hassan S. The role of inflammation and infection in preterm birth. Semin Reprod Med. 2007;25:21-39. https://doi.org/10.1055/s-2006-956773.

21. Gonik B, Cotton D. The use of amniocentesis in preterm premature rupture of membranes. Am J Perinatol. 1985;2:21-4. https://doi. org/10.1055/s-2007-999905.

22. Romero R, Yoon BH, Mazor M, Gomez R, Gonzalez R, Diamond MP, et al. A comparative study of the diagnostic performance of amniotic fluid glucose, white blood cell count, interleukin-6, and gram stain in the detection of microbial invasion in patients with preterm premature rupture of membranes. Am J Obstet Gynecol. 1993;169:839-51. https://doi. org/10.1016/0002-9378(93)90014-A.

23. Gauthier DW, Meyer WJ, Bieniarz A. Correlation of amniotic fluid glucose concentration and intraamniotic infection in patients with preterm labor or premature rupture of membranes. Am J Obstet Gynecol. 1991;165:1105-10. https://doi.org/10.1016/00029378(91)90480-F.

24. Coultrip LL, Grossman JH. Evaluation of rapid diagnostic tests in the detection of microbial invasion of the amniotic cavity. Am J Obstet Gynecol. 1992;167:1231-42. https://doi.org/10.1016/S00029378(11)91694-9.

25. Curtis M, Riggs J, Blanco J. Declines in Patient Volume: An obstetrics and gynecology teaching program's response. Am J Perinatol. 1998;15:173-6. https://doi. org/10.1055/s-2007-993920.

26. Epstein FH, Goldenberg RL, Hauth JC, Andrews WW. Intrauterine infection and preterm delivery. $\mathrm{N}$ Engl J Med. 2000;342:1500-7. https://doi.org/10.1056/ NEJM200005183422007.

27. Yoon BH, Jun JK, Park KH, Syn HC, Gomez R, Romero R. Serum C-reactive protein, white blood cell count, and amniotic fluid white blood cell count in women with preterm premature rupture of membranes. Obstet Gynecol. 1996;88:1034-40. https://doi.org/10.1016/S0029-7844(96)00339-0.

28. Oyelese Y, Vintzileos AM. The uses and limitations of the fetal biophysical profile. Clin Perinatol. 2011;38:4764. https://doi.org/10.1016/j.clp.2010.12.008.

29. Gotsch F, Romero R, Kusanovic JP, Mazaki-Tovi S, Pineles $\mathrm{BL}$, Erez O, et al. The fetal inflammatory response syndrome. Clin Obstet Gynecol. 2007; 50:652-83. https://doi.org/10.1097/GRF.0b013e31811ebef6.

30. Vintzileos AM, Campbell WA, Nochimson DJ, Connolly ME, Fuenfer MM, Hoehn GJ. The fetal biophysical profile in patients with premature rupture of the membranes-an early predictor of fetal infection. Am J Obstet Gynecol. 1985;152:510-6. https://doi. org/10.1016/0002-9378(85)90617-9.

31. Vintzileos AM, Bors-Koefoed R, Pelegano JF, Campbell WA, Rodis JF, Nochimson DJ, et al. The use of fetal biophysical profile improves pregnancy outcome in premature rupture of the membranes. Am J Obstet Gynecol. 1987;157:236-40. https:/doi.org/10.1016/ S0002-9378(87)80141-2. 
32. Heatley MK. Haines \& Taylor Obstetrical and Gynaecological Pathology, 5th edition. The Obstetrician \& Gynaecologist. 2005;7:218. https:// doi.org/10.1576/toag.7.3.218.27108.

33. Prophet EB, Instituto de las Fuerzas Armadas (Washington). Métodos histotecnológicos; 1995. p. 280.

34. Holzman C, Lin X, Senagore P, Chung H. Histologic chorioamnionitis and preterm delivery. Am J Epidemiol. 2007;166:786-94. https://doi. org/10.1093/aje/kwm168.

35. Nowak M, Oszukowski P, Szpakowski M, Malinowski A, Maciołek-Blewniewska G. [Intrauterine infections. I. The role of C-reactive protein, white blood cell count and erythrocyte sedimentation rate in pregnant women in the detection of intrauterine infection after preliminary rupture of membranes]. Ginekol Pol. 1998;69:615-22.

36. Newton ER. Preterm labor, preterm premature rupture of membranes, and chorioamnionitis. Clin Perinatol. 2005;32:571-600. https://doi.org/ 10.1016/j.clp.2005.05.001.

37. Hameed C, Tejani N, Verma UL, Archbald F. Silent chorioamnionitis as a cause of preterm labor refractory to tocolytic therapy. Am J Obstet Gynecol. 1984;149:726-30. https://doi.org/10.1016/00029378(84)90111-X.

38. Wu HC, Shen CM, Wu YY, Yuh YS, Kua KE. Subclinical histologic chorioamnionitis and related clinical and laboratory parameters in preterm deliveries. Pediatr Neonatol. 2009;50:217-21. https://doi.org/10.1016/ S1875-9572(09)60066-8.
39. Le Ray I, Mace G, Sediki M, Lirussi F, Riethmuller $\mathrm{D}$, Lentz N, et al. Changes in maternal blood inflammatory markers as a predictor of chorioamnionitis: A prospective multicenter study. Am J Reprod Immunol. 2015;73:79-90. https://doi.org/10.1111/aji.12323.

40. Curtin WM, Katzman PJ, Florescue H, Metlay LA, Ural SH. Intrapartum fever, epidural analgesia and histologic chorioamnionitis. J Perinatol. 2015;35:396400. https://doi.org/10.1038/jp. 2014.235.

41. Curtin WM, Katzman PJ, Florescue H, Metlay LA. Accuracy of signs of clinical chorioamnionitis in the term parturient. J Perinatol. 2013;33:422-8. https:// doi.org/10.1038/jp.2012.135.

42. Heller DS, Rimpel LH, Skurnick JH. Does histologic chorioamnionitis correspond to clinical chorioamnionitis? J Reprod Med. 2008;53:25-8.

43. Salafia CM, Ghidini A, Sherer DM, Pezzullo JC. Abnormalities of the fetal heart rate in preterm deliveries are associated with acute intra-amniotic infection. Obstet Gynecol Surv. 1999;54:93-4. https:// doi.org/10.1097/00006254-199902000-00008.

44. Sherer DM, Spong CY, Salafia CM. Fetal breathing movements within 24 hours of delivery in prematurity are related to histologic and clinical evidence of amnionitis. Am J Perinatol. 1997;14:337-40. https:// doi.org/10.1055/s-2007-994156.

45. Ghidini A, Salafia CM, Kirn V, Doria V, Spong CY. Biophysical profile in predicting acute ascending infection in preterm rupture of membranes before 32 weeks. Obstet Gynecol. 2000;966:201-6. https://doi. org/10.1016/S0029-7844(00)00908-X. 